Preprint of: Handbook of Research Methods and Applications in Experimental Economics, edited by Arthur Schram and Aljaz Ule, Edward Elgar Publishing, 2019, chapter 21, 401-418.

\title{
COMMUNICATION IN LABORATORY EXPERIMENTS
}

\author{
Jordi Brandts ${ }^{1}$ David J. Cooper ${ }^{2}$ and Christina Rott ${ }^{3}$ \\ "Without knowing the force of words, it is impossible to know more." \\ - Confucius
}

\begin{abstract}
In this chapter we present a first survey of laboratory studies using communication. We first discuss a number of issues about the implementation of communication in the laboratory. In particular we discuss variations in the channels, the structure and the content analysis of communication. Second, we survey a number of studies in which the effects of free-form communication are compared to those with more restricted communication. We finish with a brief reference to the question why communication matters, to what the mechanisms through which communication affects behavior are.
\end{abstract}

\footnotetext{
${ }^{1}$ Instituto de Análisis Económico (CSIC) and Barcelona GSE.

${ }^{2}$ Florida State University and University of East Anglia.

${ }^{3}$ Maastricht University.
} 


\section{INTRODUCTION}

This chapter has two distinct parts. In Sections 2, 3 and 4 we discuss a number of technical dimensions pertaining to the implementation of communication in laboratory experiments. ${ }^{4} \mathrm{In}$ Section 5 we present a selective literature review of experimental studies in which free-form communication is directly compared with more structured communication. Throughout the chapter we describe the results of what we think is important work and we do not give recommendations about whether we consider some procedures superior to others. We believe that in most cases there is just not enough evidence to make an informed judgement.

The implementation of communication in laboratory experiments can be done in many ways. We begin our chapter by discussing some central implementation issues and organize them in terms of three dimensions: channel, structure, content. The choice of these particular dimensions may be somewhat arbitrary, but we see this classification as a useful organizing tool. Our emphasis is on structure and content. In our discussion of content we pay particular attention to the issue of how to code verbal communication. An overview of the issues that we will discuss within each dimension can be found in Table 1.

\begin{tabular}{|c|c|c|}
\hline CHANNEL & STRUCTURE & CONTENT \\
\hline Paper-and-pencil & Direction & Signal \\
\hline Chat & Order & Pre-formulated \\
\hline Audio & Frequency & Free-form \\
\hline Video & & \\
\hline Face-to-face & & \\
\hline
\end{tabular}

Table 1: Dimensions of communication

\footnotetext{
${ }^{4}$ We do not cover communication in field experiments.
} 


\section{CHANNELS OF COMMUNICATION}

Possible channels of communication in laboratory experiments are paper-and-pencil messages, chat, audio, video, or face-to-face interactions. The following is an incomplete reference list. Paper-and-pencil (Charness and Dufwenberg 2006) and chat messages (Brandts, Charness, and Ellman, 2016; Brandts, Rott, and Sola, 2016) are sent in written form, whereas audio (Greiner et al. 2012), video (Brosig et al. 2003; Greiner et al. 2012), and face-to-face messages (Isaac and Walker 1988; Bochet et al. 2006; Schram et al. forthcoming) are usually exchanged orally. In faceto-face communication, participants meet physically and verbally communicate with each other (for instance, Isaac and Walker, 1988).

The main drawback of audio, video and face-to-face communication is that typically they do not allow for (complete) anonymity, which entails that the interaction between participants does not necessarily stop at the end of the experiment. ${ }^{5}$ An additional limitation of face-to-face communication is the difficulty of creating a record of what has been said. By contrast, chat, audio, and video messages can be recorded, transmitted and analyzed at a later stage (for instance, to a subsequent generation as in Chaudhuri et al., 2009).

Paper-and-pencil as well as audio and video communication need to be transcribed in order to be used. In addition, in audio and video communication one has to take into account that their content is very rich, including intonation and emphasis in audio and facial expression and body language in video, aspects that are hard to study. Brosig et al. (2003), Bochet et al. (2006), Greiner et al. (2012), as well as Greiner et al. (2014) compare the effect of different channels on outcomes in laboratory experiments.

Computer chat is becoming the most commonly used communication channel in economic laboratory experiments. Experimental software programs like z-tree (Fischbacher 2007) and oTree (Chen et al. 2016), as well as the programming language Python allow for chat communication. In

\footnotetext{
${ }^{5} \mathrm{He}$ et al. (2017) present results from experiments in which they systematically study the effects of face-to-face communication, apart from the dynamic effect just mentioned. They find that the $40 \%$ increase in cooperation in a social dilemma are mostly due to the possibility of distinguishing cooperative people from non-cooperative people ('typer recognition') and that the creation of social ties plays no role.
} 
a computerized experiment, a chat window opens (or participants have the option to open it) and participants can type in text messages. ${ }^{6}$

\section{STRUCTURE OF COMMUNICATION}

With the term 'structure' we refer here to the arrangements of who can communicate to whom, when, in what order, and how often. All these elements can be tightly controlled in the laboratory and this control allows for many potentially interesting variations. This makes it possible to study the general phenomenon of 'communication' in a very systematic way. We organize our discussion of structure into three subsections: network and direction, frequency/repetition, and order and timing.

\subsection{COMMUNICATION NETWORK AND DIRECTION}

In experiments involving only two participants the only issue is whether they both can communicate with the other participant or whether only one of the two can (and who it is). In experiments with more than two participants, there are even more possibilities, since now there are several options both with respect to who can talk to whom and who can listen to whom. The structure of these connections is what we refer to as communication network. Choi and Lee (2014), Grandjean et al. (2017) and Brandts et al. (2018) are recent experimental studies that vary the communication networks to see how it affects outcomes.

Direction of communication refers to whether communication flows one-way, two-way, or multiple-ways. The choice between these options should respond to what kind of situation one wants to represent; for example, in some organizational contexts it makes sense to consider that management can send messages to workers and that workers do not have the same possibilities.

In one-way communication, one participant in the experiment can send messages to another participant or to a group of participants who cannot reply. Messages can be transmitted in written form (for instance, Koukoumelis et al. 2012; Brandts, Rott, and Sola, 2016) or orally. Ellingsen

\footnotetext{
${ }^{6}$ Written messages might be exchanged through other channels; for instance, emails as in Bolton et al. (2003).
} 
and Johannesson (2004) and Andreoni and Rao (2011) allow players in different roles (namely buyers versus sellers, dictators versus receivers) to send one-way messages to one another.

In two-way communication messages are sent back and forth between participants or groups of participants (Cooper and Kühn 2014). Brosig et al. (2003) compare the effects of one versus two-way communication on cooperation, Cooper et al. $(1989,1992)$ on outcomes in coordination games, and Cooper and Kagel (2016) on learning to play strategically in signaling games. Multiple-way communication differs from two-way communication only in that more than two individuals are involved and allowed to send messages to the others (for instance, Murnighan and Roth 1980; Isaac and Walker 1988; Bolton et al. 2003; Irlenbusch et al. 2003). Many variations of multiple-way communication are possible. For example, in Isaac and Walker (1988) participants in an experiment on the voluntary contribution mechanism to a public good could, after each experimental rounds, openly talk face-to-face about the experimental situation.

\subsection{COMMUNICATION FREQUENCY/ REPETITION}

Communication can be implemented one-time or repeatedly. In both cases, the chronological allotment in the experiment depends on the research question. Koukoumelis et al. (2012) allow non-binding communication once at the beginning of the experiment (that is, before the participants make payoff-relevant decisions) and compare subsequent contributions to a public good to cooperation in a baseline without communication. Communication can also take place at a later stage of the experiment if, for instance, the impact of communication in a situation created through the interaction of individuals in the laboratory is the focus of research. Brandts, Rott, and Sola (2016) study, for instance, how communication affects contributions after cooperation failure in the group. In repeatedly played games communication can be repeated, that is, the same participants communicate in the same way at least twice during the experiment. The distance between the communication stages is an important aspect and depends on the research question. Is communication considered a natural every day interaction between team members? Or is communication used as an infrequent leadership tool? For example, participants converse in every round of the repeatedly played public goods game with communication in Isaac and Walker (1988), while the leader communicates with the followers only twice in the 36 rounds of the repeatedly played public goods game in Brandts, Rott, and Sola (2016). 


\subsection{COMMUNICATION ORDER}

Two- or multiple-way communication can be simultaneous or sequential. All communication channels are suitable for both simultaneous and sequential communication. Under simultaneous communication, participants read or see each other's message only after having recorded their own message for the other participant(s) as in Cooper et al. $(1989,1992)$ or Palfrey and Rosenthal (1991). Chat rooms are less structured and allow participants to write text messages that are exchanged instantaneously and in continuous time. In chat rooms as well as with voice, or video connections, in which participants talk to each other 'live' there is no particular order with respect to which participants can send messages at a particular time. Communication is unordered and interrupting others is possible (for instance, Forsythe et al. 1991; Roth, 1985, 1987; Brandts and Cooper 2007; Brandts, Charness, and Ellman, 2016). Of course, a strict order of communication can be enforced in chat, too.

\section{CONTENT OF FREE-FORM COMMUNICATION}

Communication content can vary from an abstract 'signal' to completely free-form communication with many intermediate possibilities. A signal can be a single number (for instance the contribution and punishment amount in a public goods game with punishment as in Bochet and Putterman, 2009; private information as in Rosaz and Villeval, 2012 or self-reported expected performance in an arithmetic task as in Reuben et al., 2014) or a single letter or word (e.g., indicating a particular action intention, Cooper et al., 1989, 1992). Another option is to use an expression, phrase or message, chosen from those that were pre-formulated by the experimenter. ${ }^{7}$

\footnotetext{
${ }^{7}$ When choosing the pre-formulation, previous research on communication in similar environments might be helpful. Garofalo and Rott (forthcoming) for instance use Andreoni and Rao (2011)'s analysis of the free-form communication in an ultimatum game as guideline for the pre-formulated phrases in their paper. Pre-formulation might be preferred over free-form communication if another aspect of communication is of interest (for instance, the messenger as in Garofalo and Rott, forthcoming) and the experimenters want to control the communication content. Brandts et al. (2015), for instance, want to avoid that participants reveal their gender through freely written messages. Preformulated messages can be transmitted through different channels, even face-to-face as for instance in Schram et al. (forthcoming).
} 
Free-form communication is possible for all channels, directions, orders etc. In paper-andpencil experiments, sheets with an assigned space for the communication content can be used. In written chats in computerized experiments, a chat window opens up or the participants open the chat window and write free-form messages (for instance, Cooper and Kagel 2005; Holm and Danielson 2005; Brandts et al. 2015; Brandts, Rott, and Sola, 2016).

Free-form communication can also be implemented via audio or video calls or face-to-face interaction; see for instance Greiner et al. (2012), Brosig et al. (2003), Isaac and Walker (1988) as well as Bochet et al. (2006). Since participants are free to write or say whatever they want, there are several norms for free-form communication in economic laboratory experiments: participants are not allowed to express threats outside the laboratory or reveal their identity (e.g., hair color, assigned seat, name); see for instance Brandts, Rott, and Sola (2016). Our experience is that participants always follow these instructions, but we can not rule out that they would refrain from identifying themselves and threatening others even in the absence of such instructions. The reason for asking participants not to express threats is that, since participants are typically located in the same room, threats could lead to some participants wanting to find out who threatened them and to disturb others. There are also some other elements of casual communication that may make unrestricted chat communication problematic. For example, in Latin and Slavic languages a message may reveal the gender of the sender and this may be undesirable from the experimenters' point of view. In this case, the instructions could include a requirement not to reveal one's gender. ${ }^{8}$ Alternatively, one could revert to structured communication which makes the revelation of the sender's gender impossible.

Whether and to what degree one limits the message space depends on the research question. For some studies, especially those examining specific theoretical predictions, the critical issues are control over what messages can be sent and precision in how to interpret these messages. Use of a limited message space (set of possible messages) is a sensible option for this type of research. For example, Blume et al. (1998) study how messages acquire meaning in Crawford-Sobel (1982) style

\footnotetext{
${ }^{8}$ In this context the issue of how to enforce certain communication rules arises. Reduced payment or exclusion from the experiment are possibilities. However, as we point above in practice participants typically follow the rules.
} 
cheap talk games. The theoretical predictions being tested depend on the size of the message space, so it is natural to control the number of available messages.

In contrast, for exploratory studies which do not test specific theoretical propositions the richness of unrestricted chat is an advantage. Belot and van de Ven (2017), for instance, study the ability of individuals to detect deception in face-to-face communication. The paper is about how experimental subjects decipher the subtle cues in what people say and how they say it. The research question would be meaningless in an environment without rich communication.

Many papers fall at neither extreme, making it less obvious whether limited communication or unrestricted chat (or possibly both as in the papers discussed below) ought to be used. Choosing a mode of communication involves weighing the pros and cons of the different approaches. At this point, this choice has to be based on good judgement, since there is not enough evidence for a more systematic approach.

\subsection{RECORDING AND CODING OF FREE-FORM COMMUNICATION}

In some cases, the effect of free-form communication is interesting as such, without the need to know anything about its content. For instance, in situations in which all participants have an incentive to free-ride, as in public good games, it is interesting to find out whether the possibility of communicating can lead to efficiency gains (see Isaac and Walker, 1988, among others). On the other hand, the communication content analysis can be used to understand and explain outcomes. It can give important insights as to why - or when - communication works (for instance, Cooper and Kagel 2005; Brandts, Rott, and Sola, 2016; Eich and Penczynski 2016). In this case, two main questions arise: what do participants communicate, and what is the relation to observed behavior of what they communicate. To answer them one needs to code participants' conversations.

Before discussing the coding of free-form communication, we need to briefly refer to the possibility that the mere recording of free-form communication may affect what participants say, because they feel monitored (see also Chapter V.5 on experimenter demand effects in this volume). 
For this reason some researchers decide against recording (for instance, Irlenbusch et al., 2003). We know of no systematic study of the impact of recording itself. ${ }^{9}$

\subsection{CODING IN ECONOMICS, PSYCHOLOGY/BUSINESS AND COMMUNICATION SCIENCES}

Coding of free-form communication consists of the conversion of human conversations into quantified information. Researchers in a variety of disciplines have worked on this issue. Content analysis techniques have been rigorously investigated and developed in communication sciences (for an introduction and guidebook see for instance Krippendorff, 2013, and Neuendorf, 2016). Das (2014) provides an overview of text analysis in finance, including techniques of text extraction, classification, and predictive analytics. For disciplines like public policy (Broniecki et al. 2017; Gurciullo and Mikjaylov 2017) or Psychology (Guetzkow 1950), the transformation of qualitative communication records into quantitative data and the analysis thereof is also an important research area. Argumentation (or argument) mining is an interdisciplinary field of research that tries to extract arguments from unstructured textual documents using computational linguistic and machine learning advances (Lippi and Torroni 2016). In experimental economics, researchers have proposed coding methods using both humans (for instance, Cooper and Kagel 2005; Houser and Xiao 2011; Eich and Penczynski 2016) and computerized techniques like machine learning (for instance, Penczynski, 2016).

\subsection{QUANTIFYING DATA OF FREE-FORM COMMUNICATION}

Mapping qualitative free-form communication into quantitative data is challenging. As early as in 1950, Harold Guetzkow, professor of political science, psychology, and sociology, pointed out the challenges of two essential steps of the coding procedure of qualitative data: unitizing and categorizing. Unitizing, defining the unit of communication, is particularly important for observational data obtained in free-answer surveys, interviews, court disputes, discussion rounds, or news media for instance. Classifying the data into categories is closely linked with the "[...]

\footnotetext{
${ }^{9}$ A difficult question is how to credibly communicate to participants that their communication is not being recorded. We do not see an easy solution to this. In the end credibility will have to be based on the experimenters' reputation for honesty.
} 
decision as to the size of the unit into which the material shall be divided before it is categorized" (Guetzkow, 1950, p. 47-48).

In laboratory experiments the experimenter structures and unitizes communication to some extent. Often communication is strictly separated from the decision stage (usually preceding it) as for instance in Isaak and Walker (1988), and Brandts, Rott, and Sola (2016). Other times, participants' decisions are integrated in the free-form communication stage as for instance in Cooper and Kagel (2005), Brandts and Cooper (2007) or Brandts et al. (2015). Communication is often restricted to a certain time interval (within an experimental round), which defines the unit for the categorization (for instance, Brandts and Cooper 2007; Brandts, Rott, and Sola, 2016; Andreoni and Rao 2011). ${ }^{10}$

\subsection{CODING CLASSIFICATIONS AND CATEGORIES}

Defining the categories of a coding is indispensable, irrespective of whether humans or software is used to code the data from experimental free-form communication. The development of a coding scheme depends on the objective. Burchardi and Penczynski (2014), Eich and Penczynski (2016), and Penczynski (2016) analyze the strategic reasoning of the participants in a beauty-contest game and therefore define the two classifications 'level of reasoning' and 'level-0 belief mean.' The classifications and the corresponding categories ('Level-0', 'Level-1', etc. for 'level of reasoning'; 'A Level-0 Belief from 100 to 76', 'A Level-0 Belief from 75 to 66', etc. for 'level-0 belief mean') are derived from a theoretical framework.

The 'content analysis' approach proposed by Cooper and Kagel (2005) is useful for wideranging coding of communication, namely when considering all possible categories (without prejudgment about which sorts of messages are important). ${ }^{11}$ In the first step, the authors (and research assistants) analyze independently (a subset of) the messages and develop categories. After double-checking the independent coding systems, a final coding scheme is defined, through a development of compromise process. An alternative procedure is proposed by Coffman and

\footnotetext{
${ }^{10}$ If a chat window is open, for example, for 5 minutes, the complete exchange during these 5 minutes is seen as one unit for coding.

${ }^{11}$ Brandts and Cooper (2007), Sutter and Strassmair (2009), Brandts et al. (2015), Hennig-Schmidt et al. (2008), and Brandts, Charness, and Ellman (2016) adopt a similar procedure.
} 
Niehaus (2018). In the context of an experiment in which buyers and sellers communicate with each other, the authors asked a group of experts to propose categories that would be most relevant for predicting the impact of communication. This procedure has the advantage of limiting data mining. Houser and Xiao (2011) propose another interesting alternative way of classifying messages by using coordination games.

A category-set is exhaustive if every unit of the communication data can be assigned to one of the pre-defined categories. This eventually requires residual categories such as "cannot tell" or "not mentioned," see Guetzkow (1950). For instance, Burchardi and Penczynski (2014), Eich and Penczynski (2016), and Penczynski (2016) use exhaustive category-sets.

\subsection{CODING BY PERSONS}

After defining the coding scheme, humans may be used to code the natural language messages. Different procedures have been used. In Bosch-Domenech et al. (2002), Charness and Dufwenberg (2006), Schotter and Sopher (2007), Kimbrough et al. (2008), and Brandts, Rott, and Sola (2016) the authors themselves code the experimental free-form messages. In Brandts, Charness, and Ellman (2016), a research assistant codes the communication content. If external coders are involved, it is important to train and instruct them carefully (for instance, by providing instructions as in Andreoni and Rao, 2011) so to reduce misunderstandings about categories as much as possible. Both practices are informative and can shed light on the channels and determinants of outcomes. Though more costly, the most common and also more neutral and appropriate procedure is to have two (for instance, Cooper and Kagel 2005; Brandts and Cooper 2007; Sutter and Strassmair 2009; Rydval et al. 2009) or more (for instance, Andreoni and Rao 2011; Houser and Xiao 2011; Burchardi and Penczynski 2014) coders work on the free-form messages independently. In most studies, coding is performed by research assitants, who are not involved in the experiment but have experience with research activities. It is important that coders are trained but not told about any hypotheses or purpose of the experiment. The goal is to have them create a numeric representation of the communication that is neither intentionally nor unintentionally biased. An exception is Eich and Penczynski (2016) who show that 43 non-incentivized, nonexpert coders recruited on Amazon Mechanical Turk achieve substantial to very good coding results, which are almost comparable to the performance of two research assistants in Burchardi 
and Penczynski (2014). Xiao and Houser (2005) and Houser and Xiao (2011) propose a payment scheme that incentivizes (independent) coders to coordinate on a coding scheme. For a reliable interpretation of the communication content, a control of consensus and consistency in coding across coders is important. The following measures capture the quality of coding across coders: (pairwise) correlations, consensus estimates like (pairwise) percentage agreement, Cohen's kappa (Landis and Koch 1977), or Krippendorff's alpha (Krippendorff 2013), which measures agreement between coders for qualitative (categorical) items, as well as consistency measures like Cronbach's alpha. In unambiguous cases, the assigned category is commonly used for the interpretation, otherwise the average rating of the different coders is used (which in that case captures the inherent ambiguity). ${ }^{12}$

According to Stemler (2004), classifier reliability measures can be roughly evaluated on the basis of consensus, consistency, and measurement estimates. Eich and Peczynski (2016, p. 6) summarize consensus and consistency measures as follows: "Consensus estimates are most relevant when it can be expected of classifiers to exactly agree on which rubric the observed message falls into. In contrast, consistency estimates reflect to which extent different behavior is indeed classified differently in a consistent way, even though not in the same absolute rubric. Consistency measures require rubrics with an underlying unidimensional construct that allows for differences in the direction of classification to be meaningful." While (pairwise) percentage agreements do not correct for the share of agreements that could be due to chance, Cohen's kappa and Krippendorff's alpha do correct.

\subsection{CODING SOFTWARE, MACHINE LEARNING}

The use of coding software and machine learning techniques is less widespread for the analysis of natural language data from economic experiments. Penczynski (2016) is to our knowledge the first to investigate the suitability of machine learning for the classification of free-form communication in laboratory experiments and shows that the classification results are promising compared to human classifications. The messages are first broken down into tokens. Tokens are strings - usually words or numbers - separated by spaces. Tokens that are not relevant for the text content, such as

\footnotetext{
${ }^{12}$ If agreement between coders is too low, a practical solution is to bring in an independent additional coder.
} 
stopwords, are removed and inflected relevant words are reduced to their stem (for instance, "team," "teams," and "teamed" are reduced to "team," Penczynski, 2016). The remaining tokens are counted and the rarely used tokens are removed. ${ }^{13}$ A learning method - for instance simple regression techniques or 'random forests' (Breiman 2001; Hastie et al. 2008) - then relates the frequency of the remaining tokens to categories. A substantial share of the dataset is used for training the model used by the learning method (in-sample), and the remaining minor share for testing the model (out-of-sample). As a result, certain words (for instance, the word "think") can be identified to be correlated with a particular behavior (for instance, the level of reasoning in the beauty-contest game, Penczynski, 2016). Coding practices in Economics for quasi-experimental (for instance, Freddi, 2017) and non-experimental (for instance, Gentzkow and Shapiro 2010; Hansen et al. 2017; Gentzkow, Kelly, and Taddy, 2017; Gentzkow, Shapiro, and Taddy, 2017; Ash et al. 2017) natural language data can provide guidance as to the use of software programs and machine learning techniques. The best practices might be transferred to the analysis of experimental free-form messages. Machine learning techniques can be particularly useful for large experimental communication datasets.

\subsection{CAUSAL LINKS BETWEEN CODING CATEGORIES AND OUTCOMES}

Relating the coded communication with outcomes in laboratory experiments or creating even a causal link between coding categories and outcomes is the final important step. The strategies subjects use for communication and their impact on outcomes depend on the context. In coordination games (for instance, Brandts and Cooper, 2007) or team play (for instance, Cooper and Kagel, 2005), individuals have an incentive to tell the truth about their choice intentions. In some strategic games participants are simply incentivized to communicate what they think is the best strategy like in Burchardi and Penczynski (2014), where participants are asked to give a written account of their reasoning processes to find out about their depth of reasoning.. In many games with asymmetric information (Charness and Dufwenberg, 2006, 2011, for instance), individuals have a monetary incentive to lie, however. The coding categories may be chosen such that the link to choices and outcomes in the experiment is meaningful. Meaning can be derived

\footnotetext{
${ }^{13}$ For instance, Penczynski (2016) takes out tokens that appear less than five times in all messages together.
} 
from standard economic theory (e.g., reasoning, strategic thinking, equilibrium play, efficiency and payoff considerations), behavioral theories (e.g., other-regarding preferences, fairness, social norms, promises, social distance and identity), or in an exploratory way (e.g., observations, emotions, and humor). ${ }^{14}$

\section{COMPARISON OF THE EFFECTS OF DIFFERENT COMMUNICATION TYPES}

In Part 2 we present a selective literature review of experimental studies in which free-form communication is directly compared with more structured communication. Here we are inspired by previous comparisons pertaining to important issues, like the comparisons of strategy vs direct response method of Brandts and Charness (2011), field vs laboratory results of Camerer (2011) and student vs expert subjects of Frechette (2016). We do not study the impact of communication compared to the absence of it. Rather, we focus on comparisons of communication protocols that allow for different content. Our concentration on comparing different types of communication allows us to discuss some insights that we now have about how communication works.

Charness and Dufwenberg (2006) examine the impact of non-binding communication in a one-shot principal-agent game. They study both communication from agent to principal and from principal to agent. They find that promises sent from agents to principals lead to higher levels of trust and efficiency than other messages. Their interpretation of these results is that the use of certain words by the agent leads to guilt aversion and can, hence be used as a commitment device. In these experiments communication is free-form; participants in the experiment write their messages on sheets of paper, which are then sent to their counterparts. This kind of communication makes it possible to compare the effectiveness in terms of efficiency of different endogenouslyemerged message content. The finding in this paper is that not all messages have the same impact.

\footnotetext{
${ }^{14}$ Ismayilov and Potters (2016) show that one has to be very cautious in claiming a causal link between a communication category and outcomes. In an experimental trust game, trustees who make a promise are more likely to act trustworthy than those who do not make a promise. However, the results of a control treatment with restricted (non-promise) communication show that the absence of promises does not decrease average cooperation rates. In this study the link between promises and trustworthiness is not causal. Promises are just more likely to be sent by cooperators than by non-cooperators.
} 
In particular messages that do not constitute a promise by an agent do not have a positive effect. The authors' interpretation is that words may affect the agent's beliefs about what the principal expects.

In a follow up paper Charness and Dufwenberg (2010) specifically address the comparison of the effects of free-form versus a certain type of structured communication, their motivation being a better understanding of the determinants of lying aversion. Using the same principal-agent game they compare the effects of a "full page of rich free-form communication" (p. 281) with that of a protocol in which agents could only choose between sending a pre-formulated promise and not sending any message. The results show that what the authors call 'bare promises', meaning pre-formulated promises, work differently from free-form messages; such pre-formulated promises either have no or only a marginal effect on behavior. The authors write: "On balance, and in comparison with the richer promises in Charness and Dufwenberg (2006), these results suggest that bare promises had substantially less effect on behavior.” (p. 283)

Lundquist et al. (2009) provide evidence of the strong(er) effect of freely formulated messages in asymmetric information games compared to pre-formulated messages. They employ a laboratory experiment to study lying (aversion) in a hiring game with one-sided private information about the applicant's skill. The authors investigate to which extent the degree of lying (i.e., the degree of promising honesty) and the size of the lie (i.e., the deviation of the lie from the truth) affect lying and whether communication leads to efficiency gains (driven by lying and guilt aversion). First, participants perform a real effort task, which determines the applicant's skill level, only known by the applicant. Each applicant is matched with one employer in a one-shot game. The employer decides whether (or not) to offer a contract, which is always beneficial for the applicant, but beneficial for the employer only if the applicant's skill is above a certain threshold level. A control treatment without communication serves as baseline for two treatments with preformulated messages (one with a strong promise that the claimed high skill level is true) and a treatment with a one-way free-form message from applicant to employer. Communication takes place in written form through the computer. While $77 \%$ and $64 \%$ of low-skill applicants claim high skills in the pre-formulated treatments, the corresponding share in the treatment with freeform communication is significantly lower at $40 \%$. Free-form messages are the only 
communication form that leads to significantly higher efficiency in terms of the applicant's and the employer's joint earnings.

Cooper and Kühn (2014) study a two period game derived from an infinitely repeated Bertrand duopoly in which players choose between low, medium and high prices. The period 1 game has a unique Nash equilibrium where both players choose low prices. The period 2 game is a coordination game with three pure strategy equilibria, in which players coordinate on the mutual choice of either low, medium, or high prices. The low prices equilibrium is the secure (maxmin) equilibrium, the medium prices equilibrium is risk dominant, and the high prices equilibrium is payoff dominant. In the two period game, mutual play of high prices in period 1 can be supported as part of a subgame perfect equilibrium if players play contingent strategies in period 2. Specifically, the high prices equilibrium should be played in period 2 if both players set high prices in period 1. Otherwise the low prices equilibrium should be played.

Cooper and Kühn (2014) find that free-form communication is far more effective than limited communication at fostering cooperation in a repeated game. A surprising source underlies this result. In both treatments, subjects frequently threaten to punish deviations from cooperative play. The difference is that punishments proposed with free chat are sufficiently harsh to make cooperation incentive compatible while with limited communication the most effective messages are, although available, not proposed. It appears that free chat gets subjects to think about the problem differently along a subtle but critical dimension.

Brandts, Charness and Ellman (2016) present experimental evidence about how communication changes the relative effectiveness of two types of contracts, rigid and fixed, when sellers choose unenforceable product quality after a possible cost shock. A flexible contract allows for a payment adjustment after the cost shock has occurred, while a rigid contract does not. They compare behavior without any communication, with chat and with structured communication. In the chat treatment buyer and seller can freely communicate over the computer with no restrictions on what to say or in what order to write messages. By contrast, under the structured communication protocol buyers were only allowed to state two numbers indicating how much they intended to add to the base price for each cost outcome. The rationale behind the use of this very limited type of communication is to be able to attribute its possible impact to a specific element, something that 
is harder in the case of chat, since people may not be precise about how much to add to the base price and may also discuss other aspects of the transaction. ${ }^{15}$ The results show that structured communication does affect behavior compared to the baseline case of no communication, but the effect is much larger with chat communication. The analysis of chat content suggests that chat allows for a more thorough clarification of post-shock transfers as well as the emergence of personal rapport which lead to higher efficiency levels.

Brandts, Rott and Solà (2016) conduct a laboratory experiment to study how a declining cooperation in a finitely repeated voluntary contribution game with a leader can be revived in an enduring way. Simply restarting the repeated game after a certain number of rounds - a simple fresh start representing the beginning of a new week or a new season - leads to an initial increase of cooperation, but also to a subsequent new decay (Andreoni 1988; Croson 1996). They compare whether higher and sustained cooperation can be achieved with three types of communication, which take place at the same time as a restart.

The first type of communication consists of a one-way free-form message sent by the leader to the followers. The second type is a message sent to all players at the moment of the restart, which contains a detailed explanation of the causes of decay together with advice about how to avoid it. The analysis of cooperation decay is based on the systematic study of such decay by Fischbacher and Gächter (2010). This kind of communication is meant to represent expert knowledge and advice as offered by consulting companies in natural environments. The third type of communication combines the two previous ones. In this case, the leader and followers receive the expert information and advice and then the leader sends a message to the followers, possibly using the expert information and advice.

The results show that leader communication with the followers is a much more effective intervention for increasing cooperation than an external expert explanation and advice based on the study by Fischbacher and Gächter (2010). The third type of intervention where leader communication and comprehension/advice are combined does not improve on leader

\footnotetext{
${ }^{15}$ Note that the structured communication protocol used in this paper does not allow for any back-and-forth between buyer and seller. A less rigid variation of this protocol could allow the seller to react to the buyer's intended transfers and make his own proposal. Furthermore, there could be additional rounds of information exchange of this kind.
} 
communication by itself. It is perhaps most surprising that the combination of the two types of communication has no additional effect over leader communication. Psychologists have extensively studied attitude change and persuasion (see Petty and Wegener 1998; Wood 2000; Bohner and Dickel 2011). Results of this research suggest that the effect of the "comprehension/advice" treatment could go both ways. On one hand, the better understanding provided by the message is 'directed to the desire for accuracy on the object' and this could lead to participants changing their attitude and contributing more (Wood 2000). In addition, persuasion effects increase when the message contains strong, cogent arguments (Wood 2000). However, there are also reasons to think that the "comprehension/advice" combination will lead to low contributions, since the reaction to it may be 'defensive' (Tycocinski et al. 1994). The information provided in the comprehension/advice message may cause distress and trigger a defensive reaction.

With respect to the content of the communication from leader to followers, the most commonly mentioned categories are the monetary benefit from cooperation and requesting conditional contribution. Some leaders also threaten to decrease their contribution if the followers do not cooperate at the same level, create a feeling of relationship closeness, and/or mention the previous decrease in cooperation and possible reasons thereof. The communication content is thus partly quite similar to the external "expert" explanation and advice given to the participants, while adding a personal nuance which could be important. Hence, one explanation for the findings in Brandts, Rott and Solà (2016) may be that what matters for cooperation is not production oriented communication, as contained in the comprehension/advice intervention and mostly in the communication following the expert explanation and advice, but people oriented communication as in the communication only intervention.

Brandts and Cooper (2017) study the "decentralization game," a coordination game designed to compare the benefits of centralized and decentralized management. The basic game is played by a central manager and two divisions. Either the central manager assigns product types to each division (centralization) or division managers choose their own product types (decentralization). Each division has a profit function that depends on local tastes, a randomly determined state of the world, and the chosen or assigned product types for the two divisions. The central manager is benevolent, earning the sum of the divisions' profits. 
In theory, there is no informational problem under decentralization and efficient play is consistent with an equilibrium. In practice, the two branches face an extremely difficult coordination problem. Under centralization, the coordination problem becomes trivial but the informational problem takes center stage. Ex ante, there is little reason to believe that it will be possible to achieve the best of all worlds, getting the branches to coordinate while taking advantage of their private information about the state of the world. In addition, it is unclear whether centralization or decentralization will yield higher total surplus to the organization.

All treatments feature 18 periods of the decentralization game, played in fixed groups of three subjects. For our purposes, we focus on six of the ten treatments in Brandts and Cooper (2018). ${ }^{16}$ These six treatments can be split into three classes, generally including a decentralization treatment (branches choose product types) and a centralization treatment (the central manager chooses both product types). The three classes vary what types of messages are possible. In the Baseline treatments, messages are only sent with centralization and consist of stripped down reports (possibly false) about the state of the world. Two limited communication treatments retain the limited message space but expand the types of messages that can be sent. In the Horizontal Communication treatment, product choice is decentralized and the two branches exchange a series of messages about which product types should be chosen. The Manager Suggestions treatment also has decentralized product choices. Prior to each period, the central manager sends the branches a menu of suggestions specifying what product types should be chosen for each state of the world. Finally, there are two chat treatments. In "Chat, Decentralization," the two branches chat prior to making decisions about product types. "Chat, Centralization" features three-way chat between the two branches and the central manager prior to the central manager choosing product types.

Several features of the results are worth noting: First, in the Baseline treatments, centralization vastly outperforms decentralization. ${ }^{17}$ Second, neither limited communication leads to much improvement. In both treatments, coordination failure remains a persistent problem. Third, with chat the branches largely solve the coordination problem under decentralization. This

\footnotetext{
${ }^{16}$ The other four treatments are variations of the Baseline treatment that either change the payoff function to make coordination easier or use a strangers matching rather than a partners matching.

${ }^{17}$ A subtle point is that a babbling equilibrium is not being played. The messages sent by the two branches are reasonably informative, but central managers make systematic errors using the information.
} 
improves performance, but the total surplus is still not larger than in the babbling equilibrium. Fourth, only with chat and centralization is total surplus significantly higher than predicted for the babbling equilibrium, as well as being significantly higher than either the "Baseline, Centralized" or "Chat, Decentralized" treatments. This is the only case in which we observe coordination and efficient use of the branches' information. Underlying this is a notable tendency of branches to voluntarily reveal their information. It is very rare for branches to lie about the state of the world. Unlike the "Baseline, Centralization" treatment, truth-telling does not appear to be strategic as it varies little with the state of the world or experience.

The strong differences between limited communication and unrestricted chat seem to be driven by two sources. First, more information can be exchanged with unrestricted chat. Even with multiple rounds of messages, branches struggle to coordinate their choices in the Horizontal Communication treatment. With chat, this difficulty vanishes. Second, information sharing changes with chat as the branches no longer lie to the central manager. This is obviously tied to a fear of being contradicted. After all, the branches can send contradictory messages in all of the treatments and disagreement is uncommon even what a branch lies in the chat treatment $(27 \%$, subject to lying). It instead appears that telling a lie is simply more difficult in the richer environment with unrestricted chat.

Ben-Ner et al. (2011) compare the effects of two types of communication in a trust game. In their single-proposal treatment, the trustor could make a proposal of sending and returning and subsequently the trustee could also make a proposal of how much to send and how much to return, which could be the same or different than the one made by the trustor. In the chat plus singleproposal treatment trustor and trustee could exchange messages in a private chat room for one minute and then made proposal and counter-proposals in the same way as in the single-proposal treatment.

The results show that either type of communication increases trusting, trustworthiness or both, but that the inclusion of verbal communication generates a higher impact. The authors offer an interesting explanation of these results. The single-proposal treatment does not allow participants to explain the numbers they sent, i.e., whether they were merely a proposal, a possibility being considered, or a behavior to which they would commit themselves. Text 
communication let subjects declare commitment, which was generally convincing to their counterparts and most often "self-committing."

In a companion paper Ben-Ner and Putterman (2009) added a third communication protocol, multiple proposals, in trust games in which binding and non-binding contracts are possible. In multiple proposals, proposals are made as in single proposals, but each of the two players can make up to three proposals. The results show that chat plus single proposals lead to higher payoffs for both players than single proposal and multiple proposals (both without chat) with no difference between the two.

\section{SUMMARY AND FINAL REMARKS}

In the first part of our chapter we have discussed a number of issues pertaining to the study of communication in the laboratory. In this part we briefly discuss studies that shed light on relevant issues, but we do not give clear specific recommendations about what choices to make. We believe that at this point there is not that much evidence to make such recommendations.

In the second part we present survey of studies and document that many studies have found free-form communication to have a different effect than structured communication. ${ }^{18}$ As discussed above structured communication can make sense if one wants to focus on particular aspects of the communication process. However, the restrictions that structured communication imposes are not innocuous. A final consideration is related to the trade-off between structured and free-form communication and external validity. Communication is a natural part of human life. People are used to talking as they try to reach agreements or solve problems. Forcing them to communicate in an unnatural fashion by the use of a limited message space pushes them out of natural patterns. This can affect the cognitive processes that get used. Hence, if there are no specific reasons not to use free-form communication like in Brandts et al. (2015) or Garofalo and Rott (forthcoming) as discussed earlier, it should be preferred over other options with limited content.

\footnotetext{
${ }^{18}$ In a study using non-laboratory data from a TV game show Belot et al. (2010) report that players who make voluntary promises cooperate much more than those who do not make voluntary promises. However, promises that arise in response to an explicit question by the presenter of the show are uninformative about behavior. The authors interpret this finding by saying that people do not want to volunteer lies but lie if they feel compelled to do so.
} 
We finish with a brief reference to the question why communication matters. In other words, what are the mechanisms through which communication affects behavior. Answering this question is beyond the scope of this chapter. A comparison of communication content analyses in different environments may yield some insights about these mechanisms. Such analysis can reveal what exactly, of the many things people say, has a positive impact in a particular situation. However, to fully understand the mechanisms by which communication works we may need to connect to studies in neuro-science. 


\section{REFERENCES:}

Andreoni, James. 1988. "Why Free Ride? Strategies and Learning in Public Goods Experiments." Journal of Public Economics, 37(3): 291-304.

Andreoni, James and Justin Rao. 2011. "The Power of Asking: How Communication Affects Selfishness, Empathy, and Altruism.” Journal of Public Economics, 95(7-8): 513-520.

Ash, Elliott, Daniel L. Chen, and Wei Lu. 2017. "Polarization of U.S. Circuit Court judges: A Machine Learning approach.” Working paper.

Belot, Michelle and Jeroen van de Ven. 2017. "How Private is Private Information? The Ability to Spot Deception in an Economic Game." Experimental Economics, 20(1): 19-43.

Belot, Michelle, V. Bhaskar and Jeroen van de Ven. 2010. "Promises and cooperation: Evidence from a TV game show." Journal of Economic Behavior and Organization, 73, 396-405.

Ben-Ner, Avner, Louis Putterman and Ting Ren. 2011. "Lavish Returns on Cheap Talk: Nonbinding Communication in a Trust Experiment." Journal of Socio-Economics 40(1): 1 - 13.

Ben-Ner, Avner, and Louis Putterman. 2009. "Trust, Communication, and Contracts: An Experiment” Journal of Economic Behavior and Organization 70(1-2): 106-121.

Blume, Andreas, Douglas V. DeJong, Young Gwan Kim, and Geoffrey B. Sprinkle. 1998. "Experimental Evidence on the Evolution of Meaning of Messages in Sender-Receiver Games.” American Economic Review, 88(5): 1323-1340.

Bochet, Olivier, and Louis Putterman. 2009. "Not just babble: Opening the black box of communication in a voluntary contribution experiment." European Economic Review, 53(3): 309-326.

Bochet, Olivier, Talbot Page, and Louis Putterman. 2006. "Communication and Punishment in Voluntary Contribution Experiments." Journal of Economic Behavior and Organization 60(1): $11-26$.

Bohner, Gerd, and Nina Dickel. 2011. "Attitudes and Attitude Change." Annual Review of Psychology, 62: 391-417. 
Bolton, Gary, Kalyan Chatterjee, and Kathleen McGinn. 2003. "How Communication Links Influence Coalition Bargaining: A Laboratory Investigation.” Management Science, 49(5): 583-598.

Bosch-Domenech, Antoni, Jose G. Montalvo, Rosemarie Nagel, and Albert Satorra. 2002. "One, Two, (Three), Infinity, ... : Newspaper and Lab Beauty-Contest Experiments.” American Economic Review, 92(5):1687-1701.

Brandts, Jordi and Gary Charness. 2011. "The Strategy versus the Direct-response Method: A First Survey of Experimental Comparisons." Experimental Economics, 14(3): 375-398.

Brandts, Jordi, Gary Charness, and Matthew Ellman. 2016. "Let's Talk: How Communication Affects Contract Design.” Journal of the European Economic Association, 14(4):943-974.

Brandts, Jordi, and David J. Cooper. 2007. "It's What You Say, Not What You Pay: An Experimental Study of Manager-employee Relationships in Overcoming Coordination Failure." Journal of the European Economic Association, 5(6): 1223-1268.

— 2018. "Truth Be Told: An Experimental Study of Communication and Centralization." Barcelona GSE Working Papers Series $n^{\circ} 1046$.

Brandts, Jordi, David J. Cooper, and Roberto A. Weber. 2015. "Legitimacy, Communication and Leadership in the Turnaround Game.” Management Science, 61(11): 2627-2645.

Brandts, Jordi, Leonie Gerhards, and Lydia Mechtenberg. 2018. "Deliberative Structures and their Impact on Voting Behavior under Social Conflict.” Barcelona GSE working paper.

Brandts, Jordi, Valeska Groenert, and Christina Rott. 2015. "The Impact of Advice on Women's and Men's Selection into Competition." Management Science, 61(5): 1018-1035.

Brandts, Jordi, Christina Rott, and Carles Sola. 2016. "Not Just Like Starting Over - Leadership and Revivification of Cooperation in Groups." Experimental Economics, 19(4): 792-818.

Breiman, Leo. 2001. "Random Forests.” Machine Learning, 45(1): 5-32. 
Broniecki, Philipp, Anna Hanchar, and Slava J. Mikhaylov. 2017. "Data Innovation for International Development: An Overview of Natural Language Processing for Qualitative Data Analysis.” arXiv preprint arXiv:1709.05563.

Brosig, Jeanette, Joachim Weimann, and Axel Ockenfels. 2003. "The Effect of Communication Media on Cooperation." German Economic Review, 4(2):217-241.

Burchardi, Konrad B., and Stefan P. Penczynski. 2014. "Out of Your Mind: Eliciting Individual Reasoning in One Shot Games." Games and Economic Behavior, 84(1): 39-57.

Camerer, Colin. 2011. "The Promise and Success of Lab-field Generalizability in Experimental Economics: A Critical Reply to Levitt and List" - papers.ssrn.com.

Charness, Gary, and Martin Dufwenberg. 2006. "Promises and Partnership." Econometrica, 74(6): 1579-1601.

Charness, Gary, and Martin Dufwenberg. 2010. "Bare Promises.” Economics Letters, 107(2): 281283.

2011. "Participation." American Economic Review, 101(4): 1211-37.

Chaudhuri, Ananish, Andrew Schotter, and Barry Sopher. 2009. "Talking Ourselves to Efficiency: Coordination in Intergenerational Minimum Effort Games with Private, Almost Common, and Common Knowledge of Advice.” Economic Journal, 119(534): 91-122.

Chen, Daniel L., Martin Schonger, and Chris Wickens. 2016. “oTree-An Open-source Platform for Laboratory, Online, and Field experiments." Journal of Behavioral and Experimental Finance, 9: 88-97.

Choi, Syngjoo, and Jihong Lee. 2014. "Communication, Coordination, and Networks." Journal of the European Economic Association, 12 (1): 223-247.

Coffman, Lucas and Paul Niehaus. 2018. "Pathways to Persuasion,” working paper.

Cooper, Russell, Douglas V. DeJong, Robert Forsythe, and Thomas W. Ross. 1989. "Communication in the Battle of the Sexes Game: Some Experimental Results." The RAND Journal of Economics, 20(4): 568-587. 
1992. "Communication in Coordination Games." Quarterly Journal of Economics, 107(2): 739-771.

Cooper, David J., and John H. Kagel. 2005. "Are Two Heads Better than One? Team versus Individual Play in Signaling Games.” American Economic Review, 95(3): 477-509.

- 2016. "A failure to communicate: an experimental investigation of the effects of advice on strategic play." European Economic Review, 82: 24-45.

Cooper, David J., and Kai-Uwe Kühn. 2014. "Communication, Renegotiation, and the Scope for Collusion.” American Economic Journal-Microeconomics, 6(2): 247-278.

Crawford, Vincent P., and Joel Sobel. 1982. "Strategic Information Transmission." Econometrica, 50(6): 1431-1451.

Croson, Rachel T.A. 1996. "Partners and Strangers Revisited.” Economics Letters, 53(1): 25-32.

Das, Sanjiv R. 2014. “Text and Context: Language Analytics in Finance.” Foundations and Trends ${ }^{\circledR}$ in Finance, 8(3): 145-261.

Eich, Theresa, and Stefan P. Penczynski. 2016. "On the Replicability of Intra-Team Communication Classification." University of Mannheim Working Paper.

Ellingsen, Tore, and Magnus Johannesson. 2004. "Is There a Hold - up Problem?" The Scandinavian Journal of Economics, 106(3): 475-494.

Fischbacher, Urs. 2007. "z-Tree: Zurich toolbox for ready-made economic experiments." Experimental economics, 10(2): 171-178.

Fischbacher, Urs and Simon Gächter. 2010. "Social Preferences, Beliefs, and the Dynamics of Free Riding in Public Good Experiments.” American Economic Review, 100(1): 541-556.

Forsythe, Robert, John Kennan, and Barry Sopher. 1991. “An Experimental Analysis of Strikes in Bargaining Games with One-sided Private Information.” The American Economic Review, 81(1): 253-278. 
Fréchette, Guillaume R. 2016. "Experimental Economics Across Subject Populations.” In The Handbook of Experimental Economics, vol 2., John H. Kagel and Alvin E. Roth (editors), Princeton University Press, 435-480.

Freddi, Eleanora. 2017. "Do People Avoid Morally Relevant Information? Evidence From the Refugee Crisis." CentER Discussion Paper Series No. 2017-034.

Garofalo, Orsola, and Christina Rott. Forthcoming. "Shifting Blame? Experimental Evidence on Delegating Communication." Management Science.

Gentzkow, Matthew, Bryan T. Kelly, and Matt Taddy. 2017. “Text as Data.” NBER working paper.

Gentzkow, Matthew and Jesse M. Shapiro. 2010. "What Drives Media Slant? Evidence From U.S. Daily Newspapers." Econometrica, 78(1): 35-71.

Gentzkow, Matthew, Jesse M. Shapiro, and Matt Taddy. 2017. "Measuring Polarization in Highdimensional Data: Method and Application to Congressional Speech.” NBER Working Paper No. 22423.

Grandjean, Gilles, Marco Mantovani, Ana Mauleon, and Vincent Vannetelbosch. 2017. "Communication Structure and Coalition-proofness - Experimental Evidence." European Economic Review, 94:90-102.

Greiner, Ben, Mary Caravella and Alvin Roth. 2014. "Is Avatar-to-Avatar Communication As Effective As Face-to-Face Communication? An Ultimatum Game Experiment in First and Second Life." Journal of Economic Behavior and Organization, 108: 374-382.

Greiner, Ben, Werner Güth, and Roi'i Zultan. 2012. "Social Communication and Discrimination: a Video Experiment." Experimental Economics, 15(3): 398-417.

Guetzkow, Harold. 1950. "Unitizing and Categorizing Problems in Coding Qualitative Data." Journal of Clinical Psychology, 6(1): 47-58.

Gurciullo, Stefano, and Slava Mikhaylov. 2017. "Detecting Policy Preferences and Dynamics in the UN General Debate with Neural Word Embeddings." arXiv preprint arXiv:1707.03490. 
Hansen, Stephen, Michael McMahon and Andrea Prat. 2017. "Transparency and Deliberation Within the Fomc: a Computational Linguistics Approach.” Quarterly Journal of Economics, https://doi.org/10.1093/qje/qjx045.

Hastie, Trevor, Robert Tibshirani, and Jerome Friedman. 2008. "The elements of statistical learning," vol. 2. Springer series in statistics Springer, Berlin.

He, Simin, Theo Offerman and Jeroen van de Ven. 2017. "The Sources of the Communication Gap," Management Science, 63(9), 2832-2846.

Hennig-Schmidt, Heike, Zhu-Yu Li, and Chaoliang Yang. 2008. "Why People Reject Advantageous Offers-Non-monotonic Strategies in Ultimatum Bargaining: Evaluating a Video Experiment Run in PR China." Journal of Economic Behavior \& Organization, 65(2): 373-384.

Holm, Hakan J., and Anders Danielson. 2005. "Fairness and Promises for Sale.” Working paper.

Houser, Daniel and Erte Xiao. 2011. "Classification of Natural Language Messages Using a Coordination Game.” Experimental Economics, 14(1): 1-14.

Irlenbusch, Bernd, Ulrike Leopold-Wildburger, Jörg Schütze, and Matthias Sutter. 2003. "The Effects of Communication in the Voting Procedure of the Stability and Growth Pact in EMU." Jahrbuch für Neue Politische Ökonomie, The political economy of institutional evolution. Holler, M. J. Kliemt, H. Schmidtchen, D. Streit, M. E. (Eds.), 31-52.

Ismayilov, Huseyn and Jan Potters. 2016. "Why do promises affect trustworthiness, or do they?." Experimental Economics, 19, 382-393.

Isaac, R. Mark, and James M. Walker. 1988. "Communication and Free-riding Behavior: the Voluntary Contribution Mechanism.” Economic Inquiry. 26(4): 585-608.

Kimbrough, Erik O., Vernon L. Smith, and Bart J. Wilson. 2008. "Historical Property Rights, Sociality, and the Emergence of Impersonal Exchange in Long-Distance Trade." American Economic Review, 98(3): 1009-1039. 
Koukoumelis, Anastasios, M. Vittoria Levati, and Johannes Weisser. 2012. "Leading By Words: A Voluntary Contribution Experiment with One-way Communication." Journal of Economic Behavior and Organization, 81(2): 379-390.

Krippendorff, Klaus. 2013. Content Analysis: An Introduction to its Methodology. Sage.

Landis, Richard J., and Gary G. Koch. 1977. “An Application of Hierarchical Kappa-type Statistics in the Assessment of Majority Agreement among Multiple Observers." Biometrics, 33(2): 363374.

Lippi, Marco, and Paolo Torroni. 2016. "Argumentation Mining: State of the Art and Emerging Trends." ACM Transactions on Internet Technology (TOIT), 16(2), 10.

Lundquist, Tobias, Tore Ellingsen, Erik Gribbe, and Magnus Johannesson. 2009. "The Aversion to Lying." Journal of Economic Behavior and Organization, 70(1-2): 81-92.

Murnighan, J. Keith, and Alvin E. Roth. 1980. "Effects of Group Size and Communication Availability on Coalition Bargaining in a Veto Game." Journal of Personality and Social Psychology, 39(1): 92-103.

Neuendorf, Kimberly A. 2016. The Content Analysis Guidebook. Sage.

Palfrey, Thomas R., and Howard Rosenthal. 1991. "Testing for Effects of Cheap Talk in a Public Goods Game with Private Information." Games and Economic Behavior, 3(2): 183-220.

Penczynski, Stefan P. 2016. "Using Machine Learning for Communication Classification." Working paper.

Petty, Richard E., and Duan T. Wegener. 1998. “Attitude Change: Multiple Roles for Persuasion Variables." In Handbook of Social Psychology, ed. Daniel T. Gilbert, Susan T. Fiske, Gardner Lindzey, pp 323-390. New York: McGraw-Hill.

Reuben, Ernesto, Paola Sapienza, and Luigi Zingales. 2014. "How Stereotypes Impair Women's Careers in Science." Proceedings of the National Academy of Sciences, 111: 4403-4408.

Rosaz, Julie, and Marie Claire Villeval. 2012. "Lies and Biased Evaluation: A Real-effort Experiment." Journal of Economic Behavior and Organization, 84(2): 537-549 
Roth, Alvin E. 1985. "Toward a focal-point theory of bargaining.” In “Game-Theoretic Models of Bargaining" (A. Roth, Ed.), Cambridge Univ. Press, Cambridge, UK.

1987. "Bargaining Phenomena and Bargaining Theory.” In “Laboratory Experimentation in Economics: Six Points of View" (A. Roth, Ed.), Cambridge Univ. Press, Cambridge, UK.

Rydval, Ondrej, Andreas Ortmann, and Michal Ostatnicky. 2009. "Three Very Simple Games and What it Takes to Solve Them." Journal of Economic Behavior and Organization, 72(1): 589601.

Schotter, Andrew, and Barry Sopher. 2007. "Advice and Behavior in Intergenerational Ultimatum Games: An Experimental Approach.” Games and Economic Behavior, 58(2): 365-393.

Schram, Arthur, Jordi Brandts, and Klarita Gërxhani. Forthcoming. "Status Ranking: A Hidden Channel to Gender Inequality under Competition," Experimental Economics.

Stemler, Steven E. 2004. "A comparison of consensus, consistency, and measurement approaches to estimating interrater reliability." Practical Assessment, Research \& Evaluation, 9(4): 1-19.

Sutter, Matthias, and Christina Strassmair. 2009. "Communication, Cooperation and Collusion in Team Tournaments-An Experimental Study," Games and Economic Behavior, 66(1): 506525.

Tycocinski, Orit, E. Tory Higgins, and Shelly Chaiken. 1994. "Message Framing, Selfdiscrepancies, and Yielding to Persuasive Messages: the Motivational Significance of Psychological Situations.” Personality and Social Psychology Bulletin, 20(1): 107-115.

Xiao, Erte, and Daniel Houser. 2005. "Emotion Expression in Human Punishment Behavior." Proceedings of the National Academy of Sciences, 102(20): 7398-7401.

Wood, Wendy. 2000. "Attitude change: Persuasion and Social Influence." Annual Review of Psychology, 51: 539-570. 